\title{
Pulmonary Arterial Hypertension in Systemic Sclerosis: Can We Predict Responders for Successful Therapy?
}

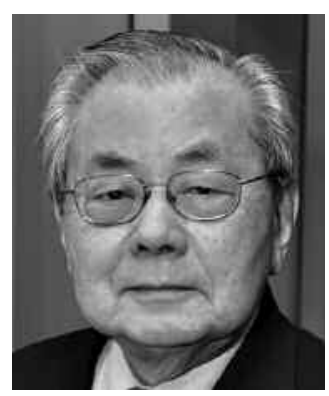

Pulmonary artery hypertension (PAH) in patients with connective tissue disease (CTD), especially systemic sclerosis (SSc), is a devastating complication that is currently the most common cause of death in these patients ${ }^{1}$. In 2003, the World Health Organization sponsored a meeting of PAH specialists to update the criteria and nomenclature for defining its different forms ${ }^{2}$. The PAH associated with SSc would now fall into the category of Group 1.3.1, which is the type associated with collagen vascular diseases, hereafter referred to as APAH. Previously, this entity was called secondary pulmonary artery hypertension. This category also includes PAH associated with congenital heart disease, portal hypertension, HIV infection, and drugs and toxins, among others (thyroid disorders, hereditary hemorrhagic telangiectasis, etc.).

Patients with SSc have an incidence of APAH ranging from $10 \%$ to $35 \%$, but those with the CREST syndrome (calcinosis, Raynaud's phenomenon, esophageal dysfunction, sclerodactyly, and telangiectasia) have a much higher incidence $(50 \%-65 \%)^{3}$. Patients with mixed CTD also have a high incidence of APAH (23\%-53\%), while those with systemic lupus erythematosus have a much lower incidence $(0.5 \%-14 \%)$. APAH is uncommon in patients with rheumatoid arthritis, Sjögren's syndrome, and dermatomyositis ${ }^{4}$.

Although the etiology of APAH is unknown, significant advances have been made in understanding the molecular abnormalities leading to the obstructive/obliterative changes within the medium-size and small pulmonary arteries, leading to the remodeling of the pulmonary circulation. Those abnormalities include the complex process of excessive cellular proliferation within the pulmonary artery vessel wall and in situ thrombosis from which microvessels and capillaries are also destroyed. The obstruction to pulmonary blood flow results in increased right-ventricular afterload (amount of increase depending upon the severity of the $\mathrm{PAH})$, right-ventricular failure in those with severe obstruction, and thereafter, premature death ${ }^{5}$.

Despite these advances in the knowledge of the molecular abnormalities associated with APAH, Michelakis and colleagues note that only 3 areas have been the subject of translational research: (1) decreased synthesis of prostacyclin, (2) downregulation of the nitric oxide/cyclic guanosine monophosphate axis, and (3) excessive synthesis of endothelin ${ }^{5}$. The article by Cella and colleagues in this issue of The Journal addresses the second and third areas outlined above ${ }^{6}$. They studied 18 patients and 18 control patients to assess and correlate levels of some plasma markers of endothelial cell activity with cardiopulmonary assessments of the right-heart and pulmonary circulation in patients with PAH associated with CTD (mainly SSc) before and after 3 months of bosentan therapy.

To my knowledge, this is the first article evaluating a comprehensive panel of plasma markers of endothelial dysfunction in patients with APAH associated with CTD. The study also makes correlations between the plasma markers and improvements in functional activity and pulmonary hemodynamics, as assessed by echocardiography. In essence, NO level was elevated before therapy with bosentan and normalized after therapy. Similarly, sCD40L (a transmembrane protein structurally related to tumor necrosis factor- $\alpha$ ) was also elevated and decreased after therapy, but remained significantly elevated above the controls. At baseline, 15 patients had significant elevations of right-ventricular systolic pressure $(50-120 \mathrm{~mm} \mathrm{Hg})$. Fourteen patients were reevaluated at 3 months: 7 showed significant reductions in right-ventricular systolic pressure (RVSP; $10-20 \mathrm{~mm} \mathrm{Hg}$ ), while the other 7 showed only modest reductions.

I find these hemodynamic results a disappointing aspect of bosentan therapy, probably indicating that bosentan does not target the precise pulmonary artery molecular abnormality causing the APAH. There was a good inverse correlation between changes in RVSP and the changes in the 6minute walk test. An interesting and perhaps important finding was the group of patients who improved after bosentan and had decreased levels of P-selectin after therapy, in contrast to those who did not respond whose Pselectin levels increased or remained elevated, suggesting

See Effect of bosentan on plasma markers of endothelial cell activity in secondary pulmonary hypertension related to CTD, page 760 
that P-selectin may be a useful predictor of beneficial longterm therapy with bosentan. Another predictor of APAH is the pulmonary diffusing capacity for carbon monoxide (DLCO). Steen and Medsger found that a low DLCO predicts development of APAH in patients with SSc on average 4.5 years before APAH is diagnosed ${ }^{1}$. However, if a patient with SSc did not have isolated APAH at the time of diagnosis of SSc, it could require up to 15 years before APAH is diagnosed. During this "incubation" period, the DLCO is progressively deteriorating. Hence, from the Pittsburgh Scleroderma Databank, we learn that almost all patients with APAH have an abnormally low DLCO, and that it relentlessly continues to deteriorate. It seems reasonable, therefore, to screen patients with SSc by means of echocardiography, pulmonary diffusing capacity, and currently, Pselectins, to identify those patients slated to develop the devastating complication of APAH. These patients should then be aggressively treated, with the goal of preventing or at least attenuating the severity of the disease.

In older studies, only pulmonary artery vasodilators have been available for treatment, but unfortunately, only a minority of patients with pulmonary hypertension were responsive ${ }^{5,7}$. The newer agents have more specific targets, and bosentan appears to be a leading agent. However, in some studies with bosentan, there was not a striking improvement in hemodynamics, and the functional improvement was not as impressive as in the present study ${ }^{8,9}$. This raises the query that although oral therapy with endothelial receptor antagonists can improve symptomatology and quality of life, can it really improve survival? It seems to this reviewer that we still have some distance to go before we can target the precise mechanism(s) causing the pulmonary arterial hypertension associated with collagen vascular diseases.

ARTHUR A. SASAHARA, MD, Senior Physician, Cardiovascular Division, Brigham and Women's Hospital, 1115 Beacon Street, Unit 12, Newton Highlands, Massachusetts 02461-1154, USA

\section{REFERENCES}

1. Steen V, Medsger TA Jr. Predictors of isolated pulmonary hypertension in patients with systemic sclerosis and limited cutaneous involvement. Arthritis Rheum 2003;48:516-22.

2. Simonneau G, Galie N, Rubin LJ, et al. Clinical classification of pulmonary hypertension. J Am Coll Cardiol 2004;43 Suppl:S5-S12.

3. Magliano M, Isenberg DA, Hillson J. Pulmonary hypertension in autoimmune rheumatic diseases: where are we now? Arthritis Rheum 2002;46:1997-2004.

4. Rubin LJ. Introduction. Diagnosis and management of pulmonary arterial hypertension: ACCP evidence-based clinical practice guidelines. Chest 2004;176:7S-9S.

5. Michelakis ED, Wilkins MR, Rabinovitch M. Emerging concepts and translational priorities in pulmonary arterial hypertension. Circulation 2008;118:1486-95.

6. Cella G, Vianello F, Cozzi F, et al. Effect of bosentan on plasma markers of endothelial cell activity in patients with secondary pulmonary hypertension related to connective tissue disease. J Rheumatol 2009;36:760-7.

7. Pietro DA, LaBresh KA, Shulman RM, Folland ED, Parisi AF, Sasahara AA. Sustained improvement in primary pulmonary hypertension during six years of treatment with sublingual isoproterenol. New Engl J Med 1984;310:1032-4.

8. Archer SL, Michelakis ED. An evidence-based approach to the management of pulmonary arterial hypertension. Curr Opin Cardiol 2006;21:385-92.

9. Mereles D, Ehlken N, Kreuscher S, et al. Exercise and respiratory training improve exercise capacity and quality of life in patients with severe chronic pulmonary hypertension. Circulation 2006;114:1482-9.

J Rheumatol 2009;36:665-6; doi:10.3899/jrheum.081322 